Casos Clínicos

Arch. Esp. Urol., 61, 3 (435-439), 2008

\section{ANGIOMIOLIPOMA RENAL CAUSANTE DE TROMBO EN CAVA Y SÍNDROME DE BUDD- CHIARI SECUNDARIO}

\section{Cristina Quicios Dorado y Antonio Allona Almagro.}

Servicio de Urología. Hospital Ramón y Cajal. Madrid. España.

Resumen.- OBJETIVO: Se aporta un caso de angiomiolipoma renal con tres peculiaridades: origen en seno renal, trombo en cava hasta ostium de aurícula derecha y presentación clínica en forma de síndrome de Budd-Chiari.

MÉTODOS: Se realiza una presentación y comentarios de las características del caso clínico.

RESULTADOS/CONCLUSIONES: El angiomiolipoma renal es un tumor benigno mesenquimal, poco frecuente (2$6.4 \%$ de los tumores renales), de predominio en el sexo femenino. Habitualmente surge en el parénquima renal, siendo infrecuente su origen en el seno renal. Puede afectar a estructuras venosas formando trombo tumoral en vena renal y en vena cava inferior, llegando incluso a aurícula derecha. Habitualmente es asintomático, constituyendo un hallazgo incidental en técnicas diagnósticas de imagen, pero su presentación clínica varía desde el dolor en flanco (lo más frecuente) hasta el TEP fatal, siendo excepcional su presentación como síndrome de Budd-Chiari. Debido al riesgo de TEP y muerte, el tratamiento quirúrgico (nefrectomía radical y trombectomía) del AML con trombo en cava está indicado aún cuando sea asintomático.

Palabras clave: Angiomiolipoma renal. Trombo en vena cava. Síndrome de Budd-Chiari.

Summary.- OBJECTIVE: We report one case of renal angiomyolipoma with three characteristics: renal sinus ori-gin, inferior vena cava tumor thrombus reaching the right atrium and Budd-Chiari syndrome.

METHODS: The characteristics of the case are presented and discussed.

RESULTS/CONCLUSIONS: Renal angiomyolipoma is a benign mesenchymal tumor. It is an uncommon tumor $(2-6.4 \%$ of all kidney fumors), with a female predominance. Angiomyolipoma most often originate from the renal parenchyma but they can rarely originate from the renal sinus. They can involve the renal vein, the inferior vena cava and even the right atrium as a tumor thrombus. Angiomyolipoma commonly present as an incidental finding on radiographic studies, but the clinical presentation varies from flank pain to fatal pulmonary tumor embolism. The Budd-Chiari syndrome is an extremely rare presentation. Because of the risk of potentially fatal cardiopulmonary embolism and death, surgical treatment (radical nephrectomy plus tumor thrombectomy) of these le-sions is indicated even when they are asymptomatic.

Keywords: Renal angiomyolipoma. Vena cava tumor thrombus. Budd-Chiari syndrome.

\section{INTRODUCCIÓN}

El angiomiolipoma (AML) renal es un tumor benigno, poco frecuente y con predominio en el sexo femenino. Debe su nombre a sus 3 componentes: células grasas, células musculares lisas y vasos sanguíneos.

Normalmente asintomático, suele ser un hallazgo al realizar técnicas de imagen abdominales por otros motivos. Su forma de presentación más frecuente es dolor en flanco y/o hematuria.

Aunque por lo general presenta un curso benigno con desarrollo local no invasivo y crecimiento lento, se han descrito variantes malignas con invasión local y metástasis a distancia. 
Por otro lado, puede afectar a la vena renal y vena cava inferior (VCl) como trombo tumoral. En este caso el tratamiento debe ser quirúrgico aún en ausencia de sintomatología debido al alto riesgo de embolismo cardiopulmonar.

Se presenta un caso de angiomiolipoma renal con tres peculiaridades: origen en seno renal, trombo en cava hasta ostium de aurícula derecha y presentación clínica en forma de síndrome de Budd-Chiari. Aunque descrito en la literatura, no se ha encontrado en la revisión realizada ningún caso de AML renal con trombo en cava y presentación como síndrome de Budd-Chiari, por lo que creemos que este es el primer caso publicado.

\section{CASO CLÍNICO}

Mujer de 41 años sin antecedentes de interés que presenta dolor abdominal difuso con mayor intensidad en epigastrio de 1 semana de evolución junto con náuseas, vómitos biliosos y mal estado general. El dolor aumenta con la tos e inspiración. No refiere fiebre ni sensación distérmica. No coluria ni acolia.

A la exploración física la paciente se encuentra consciente y orientada, afectada por el dolor e impresiona de gravedad. Palidez mucocutánea. Paciente afebril y normotensa. Auscultación cardiopulmonar sin hallazgos. Abdomen sin masas ni megalias, con dolor abdominal generalizado a la palpación, más intenso en epigastrio, sin signos de irritación peritoneal (Murphy negativo). No se aprecia circulación colateral. No edemas en miembros inferiores. No signos de trombosis venosa profunda (TVP).

Analíticamente presenta bioquímica normal con aumento de transaminasas (GOT: $146 \mathrm{U} / \mathrm{L}, \mathrm{GPT}: 171 \mathrm{U} / \mathrm{L}$ ). Hemograma con hemoglobina: $11.4 \mathrm{~g} / \mathrm{dL}$, hematocrito: $34 \%$, leucocitos: 12700 sin neutrofilia y plaquetas: 291000 . Hemostasia con INR: 1.3 y tiempo de cefalina $53.3 \mathrm{seg}$.

Se realiza TC (Figura 1) donde se observa trombo con densidad grasa que se extiende desde riñón izquierdo a vena renal izquierda (VRI), vena cava inferior (VCl) y ostium de aurícula derecha. Venas suprahepáticas libres. No oclusión completa de la luz de $\mathrm{VCl}$. Anormalidad inespecífica de cabeza de páncreas.

Debido a este último dato del TC junto con la intensa epigastralgia se decide realizar ECO abdómino-pélvica encontrando: hígado y vía biliar intra y extrahepática normales. Lipoma en $\mathrm{VCl}$. Signos ecográficos de colecistitis, probablemente perforada y posible pancreatitis. Líquido libre en pelvis, espacios perihepático, periesplénico y perirrenal derecho (en mayor cuantía que en TC previo).

Se plantea diagnóstico diferencial entre patología bilio-pancreática vs. Síndrome de Budd-Chiari por lo que se decide realizar segundo TC (Figura 2) apreciando derrame pleural bilateral, engrosamiento de pliegues gástricos con aumento de la captación de contraste y angiomiolipoma $(\mathrm{AML})$ vs. lipoma renal que se extiende hacia $\mathrm{VCl}$ y ostium de la aurícula derecha, con paso de contraste en todo el recorrido de las estructuras venosas, con vena porta y suprahepáticas permeables. Existen imágenes sugestivas de congestión hepática: hepatomegalia, aumento de calibre de vena porta y suprahepáticas con retardo e la llegada de contraste a las mismas, edema periportal, bazo y páncreas atigrado y abundante líquido libre.

Con diagnóstico de presunción de síndrome de BuddChiari secundario a trombo en cava se realiza paracentesis bajo control ecográfico, con el fin de excluir con mayor certeza proceso peritoneal infeccioso/ tumoral como causante de dicho sín drome. Se obtiene resultado analítico de trasudado descartando proceso peritoneal. En ecografía transesofágica la paciente presenta buena función ventricular sin trombo intracavitario, estando la tricúspide libre y existiendo derrame pericárdico.

Finalmente, con diagnóstico de Síndrome de Budd-Chiari secundario a trombo en cava (AML vs. Lipoma), y ante empeoramiento clínico de la paciente con mal estado general, se decide intervención quirúrgica urgente a las 48 horas de su llegada al Servicio de Urgencias.

INTERVENCIÓN QUIRÚRGICA. Se trata de un trombo con nivel quirúrgico IV al presentar situación supradiafragmática llegando al ostium de aurícula derecha. Se realiza incisión tipo Chevron y acceso a retroperitoneo mediante apertura desde asa fija hasta hiato de Wins-

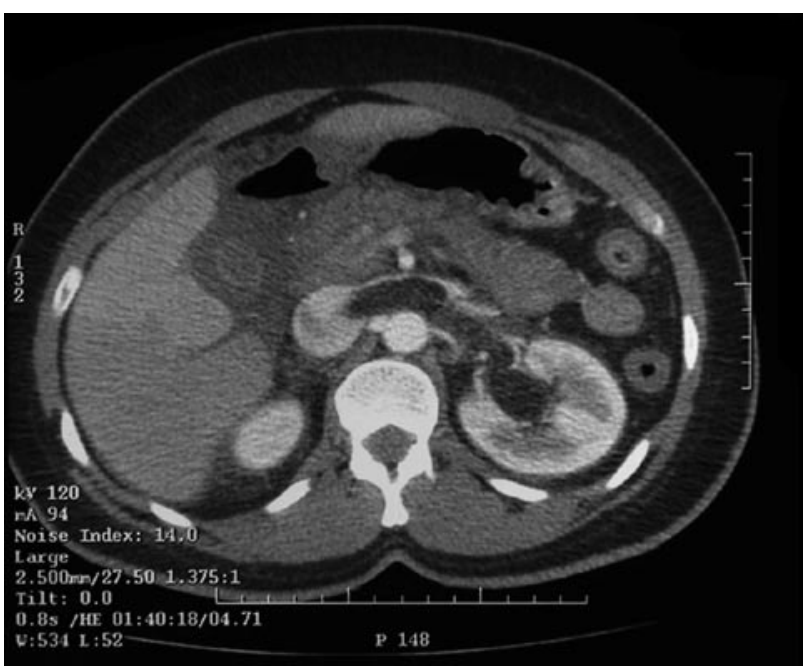

FIGURA 1. TAC. Trombo con densidad grasa. Anormalidad inespecífica de cabeza pancreática. 
low para permitir completa liberación ascendente del paquete intestinal, incluyendo ligadura de vena mesentérica inferior. La movilización hepática se logra seccionando los ligamentos redondo, coronario, triangulares derecho e izquierdo y falciforme. A continuación se libera el riñón izquierdo y su hilio correspondiente, comenzando luego con la liberación de la vena cava, empezando a nivel infrarrenal y ascendiendo hasta nivel suprahepático con control de vena renal derecha (VRD) y sección del resto de ramas excepto venas suprahepáticas que se comprueban libres de trombo tumoral y son las únicas que fijan la cava al hígado. Se amplía el hiato diafragmático y se exprime el trombo hasta nivel infradiafragmático. Se realiza clampaje de pedículo hepático (maniobra de Pringle), de VCl proximal y distal y VRD con posterior cavotomía y apertura VRI, extracción completa del trombo y nefrectomía izquierda (Figura 3).

ANATOMÍA PATOLÓGICA: angiomiolipoma de predominio lipomatoso del seno renal con infiltración vascular.

\section{DISCUSIÓN}

El angiomiolipoma (AML) renal es un tumor benigno mesenquimal formado por células grasas, células musculares lisas y vasos sanguíneos. Es infrecuente, representando entre $2-6.4 \%$ de todos los tumores renales, con predominio en el sexo femenino (1-3).

Habitualmente asintomático, suele ser un hallazgo casual en cualquier tipo de estudio radiológico realizado por otras causas. Los síntomas más frecuentes de presentación son el dolor en flanco y la hematuria, siendo los

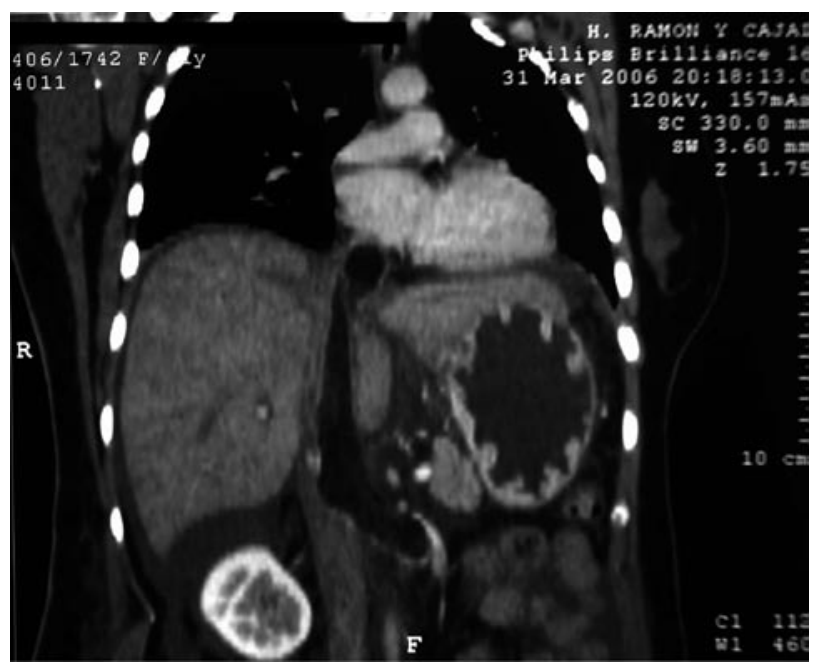

FIGURA 2. TAC body: trombo con densidad grasa que se extiende desde riñón izquierdo hasta ostium de aurícula derecha. Signos de congestión hepática. tumores de mayor tamaño los que tienen más probabilidad de ocasionar síntomas y cuadros de hemorragia espontánea, necesitando por tanto tratamiento (1).

Aparece en dos situaciones diferentes: de forma esporádica ó formando parte del síndrome de Esclerosis Tuberosa. Aunque el AML es infrecuente en la población general, el $80 \%$ ocurren en pacientes sin Esclerosis Tuberosa, frecuentemente como tumor renal solitario. El AML asociado a Esclerosis Tuberosa suele ser multicéntrico y bilateral, con presentación a edades más tempranas. Son de mayor tamaño y causantes de hemorragia de forma más frecuente que en los casos esporádicos, con mayor tendencia a crecer y a necesitar cirugía (1).

Por lo general estos tumores presentan curso benigno, con un desarrollo local no invasivo y con un crecimiento lento (1-3). Existen casos de degeneración sarcomatosa con invasión local y metástasis, descritos como transformación maligna de un AML previo benigno. También se ha descrito el AML epitelioide como una variante maligna $(2,3)$.

El AML tiene su origen en el parénquima renal, aunque también puede surgir de la cápsula renal o bien ocurrir en otros órganos abdominales como hígado, bazo, trompa de Falopio y tejido linfático. Raramente se origina a partir del seno renal como ocurre en el caso presentado (4). El TC y la RMN gracias a su capacidad para detectar pequeña cantidad de grasa pueden diagnosticar AML del parénquima, pero tienen capacidad limitada en el diagnóstico del AML circunscrito al seno renal. En los casos de AML del seno renal sin trombo asociado, es importante diferenciarlo de otras masas del seno que contienen grasa como lipomatosis, lipoma y liposarcoma, ya que el manejo terapéutico es diferente. La mayoría de estos casos descritos en la literatura se han diagnosticado tras exploración quirúrgica aunque Metro y cols. (4) abogan por la biopsia percutánea como alternativa diagnóstica.

Aunque es raro su crecimiento extarrenal e invasión vascular, el AML puede presentarse con trombo en vena renal, vena cava inferior e incluso en aurícula derecha, existiendo múltiples casos publicados $(1,5)$. La invasión de vena renal y $\mathrm{VCl}$ por $\mathrm{AML}$ renal fue descrita por primera vez por Kutcher y cols. (6). Su presentación clínica varía desde la forma asintomática (más frecuente), suponiendo un hallazgo incidental, hasta el embolismo pulmonar fatal.

Una forma de presentación clínica de trombo en cava secundario a $A M L$ descrita en la literatura es como síndrome de Budd-Chiari. El síndrome de Budd-Chiari se produce como consecuencia de la obstrucción del flujo venoso hepático por oclusión de las venas suprahepáticas o de la vena cava inferior en su segmento suprahepático (7). Clínicamente se distingue una forma aguda $(90 \%)$ caracterizada por dolor abdominal, vómitos, hepatomegalia dolorosa y ascitis que puede desembocar, 
en caso de obstrucción completa, en insuficiencia hepática aguda grave con encefalopatía, Coagulación Intravascular Diseminada (CID) y muerte. Existen también las formas subaguda y crónica con aparición progresiva de un síndrome de hipertensión portal (esplenomegalia, ascitis, varices esofágicas y hepatomegalia dolorosa). La circulación colateral y el edema en miembros inferiores pueden aparecer en los casos asociados a trombo en $\mathrm{VCl}(8)$.

Entre las numerosas causas de este síndrome (estados de hipercoagulabilidad, estrógenos, etc.) las menos frecuentes son las tumorales hepáticas ó renales (tumor de Wilms, liposarcoma, rabdomiosarcoma, CCR). Ante la sospecha de Budd-Chiari la prueba diagnóstica de elección es el TC (8) (imagen típica de estancamiento del medio de contraste e hígado grande y congestivo), que además aportará información de la causa del síndrome. En el caso de duda diagnóstica será necesaria la realización de paracentesis: la obtención de exudado orienta hacia proceso peritoneal primario (infección, tumor) y el trasudado hacia alteración de función hepática (cirrosis, hepatitis viral) ó congestión hepática (Síndrome de Budd- Chiari, pericarditis constrictiva, ICC).

Es importante destacar que aunque existen en la literatura casos descritos de AML renal con trombo en cava, la mayoría son asintomáticos o bien se presentan con dolor en flanco. No hemos encontrado en la revisión realizada ningún paciente con síndrome de Budd Chiari secundario a $A M L$ con trombo en cava, por lo que creemos que este es el primer caso publicado de estas características. Sí existen casos descritos de Budd- Chiari secundario a CCR con trombo en $\mathrm{VCl}$ (8-10). Así, Kume y cols. (8) analizan 12 pacientes con CCR y trombo en cava ocasionando este síndrome, 7 de los cuales

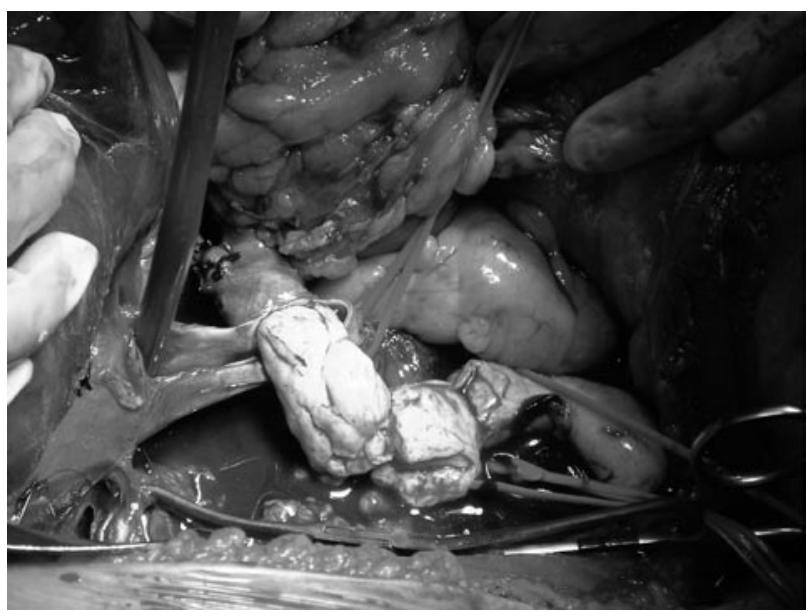

FIGURA 3. Extracción del trombo de vena cava inferior tras clampaje y cavotomía. A continuación se procede a vaciamiento de vena renal izquierda. se presentan de forma aguda. Afirman que el síndrome en sí mismo no afecta al pronóstico y que su presencia, - la de fallo hepático no debe contraindicar la cirugía $(8,11)$. Fujita y cols. (12) describen un caso de rabdomiosarcoma de $\mathrm{VCl}$ con Budd- Chiari secundario y $\mathrm{Ke}$ lleher y cols. (13) un AML hepático causante de dicho síndrome.

En cuanto al diagnóstico por imagen, el AML renal se puede diagnosticar con relativa facilidad al observar grasa en las diferentes técnicas de imagen radiológicas. Se trata de le neoplasia renal más hiperecogénica en la ecografía. El diagnóstico se confirma con TC, usando la RMN cuando el TC no es concluyente ó en determinados casos para planificar una intervención quirúrgica. Sin embargo, en el caso de AML renal con trombo en cava la RMN representa la técnica de imagen con mayor grado de fiabilidad para determinar la extensión craneal del trombo y establecer la invasión de la pared vascular por el tumor siendo fundamental a la hora de plantear la intervención (1 1). En estos casos se considera necesario un completo examen radiológico con TC y RMN cuya asociación tiene un valor predictivo del $100 \%$ (1 1). En el caso presentado no fue posible la realización de RMN al tratarse de una intervención urgente. La ecografía transesofágica pre e intraoperatoria es útil para confirmar la extensión y movilidad del trombo.

El tratamiento en el AML renal está determinado por los síntomas y el tamaño tumoral. Así en los tumores asintomáticos y menores de $4 \mathrm{~cm}$. el manejo es observación con ECO/TC anual para vigilar el crecimiento. Deben tratarse en caso de sospecha de malignidad, hemorragia espontánea que causa clínica, hematuria y riesgo de rotura.

\section{CONCLUSIÓN}

Debido al riesgo de TEP y muerte, el tratamiento quirúrgico (nefrectomía radical y trombectomía) del $\mathrm{AML}$ con trombo en cava está indicado aun cuando sean asintomáticos $(1,11)$, aconsejando embolización previa para disminuir riesgo de sangrado. La técnica quirúrgica está basada en los exámenes radiológicos siendo determinante para su planificación el nivel que alcanza el extremo superior del trombo tumoral en el interior de vena cava.

Hay que considerar siempre que debe evitarse en lo posible tener que proceder a la apertura del tórax y a la instauración de circulación extracorpórea, ciñéndola a los raros casos en que es estrictamente necesaria, pues conlleva anticoagulación del paciente y un alto riesgo de sangrado. En la mayoría de los casos, aplicando las técnicas de liberación hepática practicadas en la realización del transplante hepático basta para realizar la cirugía, tal como ocurre en el caso presentado. 


\section{BIBLIOGRAFÍA y LECTURAS RECOMENDADAS (*lectura de interés $y^{* *}$ lectura fundamental)}

**1. NELSON, C.P.; SANDA, M.G.: "Contemporary diagnosis and management of renal angiomyolipoma". J. Urol., 168: 1315, 2002.

2. SERRANO FRAGO, P.; DEL AGUA ARIAS CAMISÓN, C.; GIL SANZ, M.J. y cols.: "Controversies related to epithelioid variant of renal angiomyolipoma: a review of the literature". Urology, 67: 846, 2006.

*3. CHRISTIANO, A.P.; YANG, W.; GERBER, G.S.: "Malignant transformation of renal angiomyolipoma". J. Urol., 161: 1900, 1999.

*4. METRO, M.J.; RAMCHANDANI, P.; BANNER, M.P. y cols.: "Angiomyolipoma of the renal sinus: Diagnosis by percutaneous biopsy”. Urology, 55: 286, 2000.

*5. BAERT, J.; VANDAMME, B.; SCIOT, R. y cols.: "Benign angiomyolipoma involving the renal vein and vena cava as a tumor thrombus: case report". J. Urol., 153: 1205, 1995.

6. DITONNO, P.; SMITH, R.B.; KOYLE, M.A. y cols.: "Renal angiomyolipomas of the perinephric space". J. Urol., 147: 447, 1992.

7. RUH, J.; MALAGO, M.; BUSCH, Y. y cols.: "Management of Budd-Chiari Syndrome". Digestive Diseases and Sciences, 50: 540, 2005.

**8. KUME, H.; KAMEYAMA, S.; KASUYA, Y. y cols.: "Surgical treatment of renal cell carcinoma associated with Budd-Chiari síndrome: Report of tour cases and review of the literature". European Journal of Surgical Oncology, 25: 71, 1999.

*9. NAKAJIMA, Y.; BABA, S.; NAGAHAMA, T. y cols.: "Renal cell carcinoma presenting as Budd-Chiari síndrome”. Urol. Int., 44: 173, 1989.

10. YANAGIZAWA, R.; KARASAWA, E.; IIIZUMI, T. y cols.: "Case of renal cell carcinoma causing Budd-Chiari síndrome by tumor thrombus of inferior vena cava". Nipón Hinyokika Gakkai Zasshi, 74: 1692, 1983.

**11. GARCÍA RTELLS, D.; FERNÉNDEZ FERNÁNDEZ, E.; DE VICENTE, E. y cols.: "Estrategia quirúrgica en el carcinoma de células renales con extensión a la circulación venosa". Actas Urol. Esp., 29: 448, 2005.

*12. FUJITA, H.; KAWATA, K.; SAWADA, T. y cols.: "Rabdomiosarcoma in the inferior vena cava with secondary Budd-Chiari síndrome”. Internal Medicine, 32: 67, 1993.

13. KELLEHER, T.; STAUNTON, M.; MALONE, D. y cols.: "Budd-Chiari síndrome associated with angiomyolipoma of the liver". Journal of Hepatology, 40: 1041, 2004.

\section{Casos Clínicos}

Arch. Esp. Urol., 61, 3 (439-441), 2008

\section{RUPTURA ESPONTÁNEA DE QUISTE RENAL SIMPLE EN EL SISTEMA PIELOCALICIAL. EVOLUCION DESDE BOSNIAK I A IIF}

\author{
Alberto Hernández Castrillo, Enrique de Diego \\ Rodríguez, Miguel Ángel Rado Velázquez y José \\ Manuel Lanzas Prieto.
}

Servicio de Urología. Hospital Comarcal de Laredo. Laredo. Cantabria. España.

Resumen.- OBJETIVO: Comunicar un caso de ruptura espontánea de quiste renal simple en el sistema pielocalicial.

MÉTODOS: Exponemos el caso de una paciente de 47 años afecta de un quiste renal simple (Bosniak I) de 17 centímetros y de una litiasis renal ipsilateral. La paciente presentó una infección urinaria acompañada de fiebre y dolor lumbar, comprobándose después mediante TAC el drenaje espontáneo del quiste a la vía urinaria.

RESULTADOS: El quiste una vez drenado, se redujo en tamaño y se calcificó. Las calcificaciones son gruesas y nodulares. Actualmente presenta un quiste clasificable como Bosniak IIF.

CONCLUSIÓN: Los quistes renales simples pueden, bajo distintas circunstancias, romperse y drenar hacia la vía urinaria.

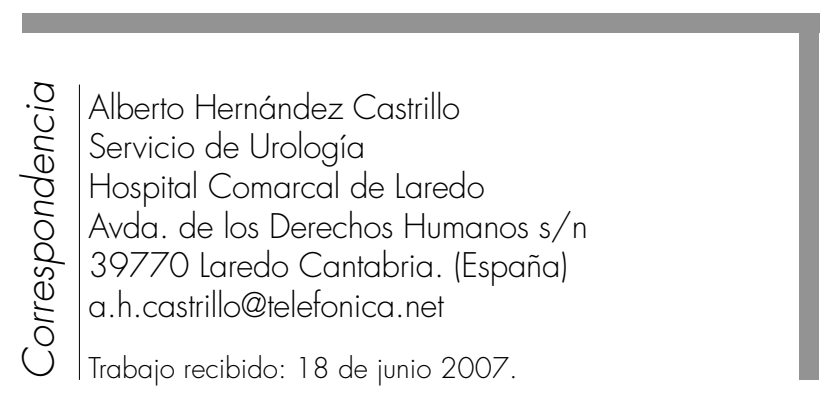

